\title{
Veinte años de erradicación del trabajo infantil en Colombia*
}

\author{
Twenty Years Eradicating Child Labour in Colombia \\ María del Pilar Jaramillo Mantilla ${ }^{1}$
}

Para citar este artícullo: Jaramillo, M. P. (2017). Veinte años de erradicación del trabajo infantil en Colombia. Infancias Imágenes, 16(1), 43-59.

\section{Resumen}

En este artículo se hace una revisión de las políticas formuladas y ejecutadas desde mediados de la década de 1990 para la erradicación del trabajo infantil en Colombia; señala sus relativos logros e impactos y su progresiva cualificación. A pesar de la reducción significativa del número de niños, niñas y adolescentes que trabajan, el problema sigue siendo muy grande. El examen de las estadísticas nacionales del DANE para los años de 2012 a 2016 evidencia tanto los logros como las limitaciones de la acción institucional. Para concluir, se sugieren posibles explicaciones a la persistencia y gravedad del problema del trabajo infantil.

Palabras clave: trabajo infantil; trabajador doméstico; derechos del niño; protección de la infancia; adolescentes; ocupación; jornada laboral; ingresos. [Tesauro Unesco: http://vocabularies.unesco.org/browser/thesaurus/es/Tesauro OIT: http://www.ilo.org/inform/online-information-resources/ terminology/thesaurus/lang--es/index.htm]
Recibido: 15-enero-2017 / Aprobado: 07-mayo-2017

\begin{abstract}
This article reviews the policies formulated and implemented since the mid-1990s for the eradication of child labour in Colombia. It indicates its relative achievements and impacts, and its progressive qualification. Despite significant reduction in the number of working children, the problem remains very serious. The review of national statistics of the National Administrative Department of Statistics for the years 2012 to 2016 evidences both achievements and limitations of institutional action. In conclusion, possible explanations for the persistence and seriousness of the problem of child labour are suggested.
\end{abstract}

Keywords: child labour; domestic workers; rights of the child; child protection; teenagers; occupations; workday; income.

\footnotetext{
* Artículo de revisión derivado de la tesis doctoral, iniciada en 2002 y culminada en 2013, El trabajo infantil. El caso de "El Prado" en Bogotá, D. C. (Colombia), avalada por la Universidad de Salamanca (España) y con financiación para 2012 y 2013 de la Universidad Distrital Francisco José de Caldas. Artículo escrito en 2016.

1 Doctora en Psicología Social y Antropología de la Universidad de Salamanca. Docente de la Facultad de Ciencias y Educación, Universidad Distrital Francisco José de Caldas. Correo electrónico: jaramillomantilla@hotmail.com
} 


\section{Introducción}

El trabajo infantil ha existido probablemente desde el origen de las sociedades. Durante siglos fue el equivalente de la escuela, el mecanismo mediante el cual los niños aprendían al lado de sus padres, o como aprendices de otros maestros, las destrezas necesarias para el desempeño de un oficio. Con el advenimiento de la Revolución Industrial y la implantación de la educación pública, cada vez fue más claro que el trabajo de los niños los perjudicaba a ellos y a toda la sociedad porque les impedía un desarrollo sano y la adquisición de los conocimientos y habilidades necesarios para desenvolverse en contextos laborales que ganaban en complejidad (Salazar, s.f.). Desde la creación de la Organización Internacional del Trabajo (OIT) en 1919, la prohibición del trabajo infantil se incorporó en la agenda pública de los gobiernos, y con la creación de la Organización de Naciones Unidas (ONU) y sus organismos vinculados como el Fondo de las Naciones Unidas para la Infancia (Unicef), la protección de los niños y la prohibición del trabajo infantil cobró cada vez mayor importancia. La Declaración de Derechos del Niño (ONU, 1959) dice, en su principio 9: "No deberá permitirse al niño trabajar antes de una edad mínima adecuada; en ningún caso se le dedicará ni se le permitirá que se dedique a ocupación o empleo alguno que pueda perjudicar su salud o su educación, o impedir su desarrollo físico, mental o moral". En las siguientes décadas se han desarrollado instrumentos normativos nacionales e internacionales en todo el mundo, como lo denota la Convención de Derechos del Niño de 1989. La cooperación de la OIT y de Unicef ha logrado incorporar en las legislaciones y políticas nacionales estrategias y programas para disminuir o erradicar el trabajo infantil.

Por otra parte, el trabajo de los menores es una realidad compleja, se da en diversos sectores productivos y oficios, e incluye modalidades tan disímiles como el trabajo en oficios domésticos, en la minería, en el comercio informal, en la agricultura e incluso en actividades ilegales y en algunos países en trabajo esclavo. Hay modalidades relativamente benignas, como podría ser la participación en oficios domésticos por tiempos breves o inclusive el trabajo remunerado en labores ligeras que no impidan estudiar, que hacen difícil fijar con exactitud el límite de lo tolerable.

En el presente texto se hace una somera revisión de los planes y políticas de alcance nacional orientados a la erradicación del trabajo infantil, desde su origen a mediados de los años noventa del siglo anterior. Examina, también, las estadísticas nacionales de los últimos cinco años para determinar los rasgos más notorios de la infancia trabajadora y sugiere algunas explicaciones a la aparente paradoja de la persistencia del trabajo infantil a pesar de la enorme cantidad y calidad de los esfuerzos orientados a su erradicación. Las fuentes de información usadas son los textos de los planes y políticas, informes estadísticos del Departamento Administrativo Nacional de Estadística (DANE) y textos de análisis de instituciones públicas nacionales o internacionales y de especialistas.

\section{Veinte años de planes y políticas para la erradicación del trabajo infantil en Colombia}

En Colombia y probablemente en otros países el progreso en el tratamiento del problema del trabajo infantil ha sido lento, a pesar de que ya cuenta con una tradición de casi un siglo de presencia en la agenda de las políticas públicas.

Un estudio llevado a cabo en 2003 por el Observatorio sobre Infancia de la Universidad Nacional de Colombia, analiza las políticas públicas hasta ese mismo año (Álvarez et al., 2003). Al reseñar el desarrollo histórico de la acción estatal, se sugiere que en principio el tema fue abordado como una cuestión de regulaciones laborales, en la órbita sectorial del Ministerio de Trabajo: garantizar el respeto de la edad mínima de vinculación al trabajo, evitar la explotación de los menores por las condiciones de trabajo, las jornadas largas o la baja remuneración. A partir de 1970, poco después de la creación en 1968 del Instituto Colombiano de Bienestar Familiar, se despierta el interés de investigadores e instituciones en conocer diversos aspectos de la situación de la infancia, entre ellos la cuestión del trabajo, pero visto en un marco más amplio de las condiciones de desarrollo de niños y niñas. 
Los estudios sobre el trabajo infanti ${ }^{2}$ y la innovación en el terreno de las políticas públicas para reducirlo, concentran mucha atención institucional en las últimas tres décadas del siglo:

Interesantes estudios sobre el trabajo infantil en Colombia, particularmente en Bogotá, desarrollan a finales de la década del setenta Muñoz y Palacios (1978) quienes destacan que los menores trabajadores, en su mayoría, se desempeñan fuera del hogar y que algunos perciben remuneración en especie, principalmente las niñas. El Departamento Nacional de Planeación (DNP) (1980) revisó la situación del trabajo infantil en el nororiente capitalino. Zambrano (1980) evidencia la realidad del trabajo infantil no remunerado y también invisibilizado tanto por parte de la familia como por la sociedad. Ayala (1982) analizó la situación de los estudiantes de primaria y su desempeño laboral, contrastando el trabajo según pertenencia a escuela pública o privada; encontrando una gran variedad de trabajos realizados por los menores en extensas jornadas y una cierta tendencia de oficios según género. Aldana (1983) indaga acerca de la participación laboral de los menores escolarizados y denota que es mayor la proporción de niñas trabajando en el hogar y en condiciones de riesgo con respecto a los niños, que trabajan fuera de la casa en el sector servicios, industria y comercio. Gutiérrez (1984) denuncia el trabajo infantil minero y aquel que se realiza en obras de construcción en donde la "ayuda" de los menores pareciera definitiva para

2 Se entenderá trabajo infantil en el texto de acuerdo con la definición de la OIT: "Todo trabajo que priva a los niños de su niñez, su potencial y su dignidad, y que es perjudicial para su desarrollo físico y psicológico. Así pues, se alude al trabajo que: es peligroso y prejudicial para el bienestar físico, mental o moral del niño; interfiere con su escolarización puesto que: les priva de la posibilidad de asistir a clases; les obliga a abandonar la escuela de forma prematura, o les exige combinar el estudio con un trabajo pesado y que insume mucho tiempo" (OIT, s.f.). Esta definición es compatible con la que usa el DANE para la medición estadística del fenómeno: "Todos los niños, niñas y adolescentes entre 5 y 17 años de edad que durante la semana de referencia participaron en el proceso de producción de bienes y servicios, en una de las siguientes situaciones: 1. Trabajaron al menos 1 hora a cambio de ingresos monetarios o en especie, o al menos una hora sin recibir pago en calidad de trabajador familiar sin remuneración o trabajador sin remuneración en empresas o negocios de otros hogares. 2. No trabajaron en la semana de referencia por vacaciones, licencias, etc., pero tenían un empleo o negocio, o estaban vinculados a un proceso de producción cualquiera y con seguridad, terminada la ausencia regresarán a su trabajo" (DANE, 2015, p. 7). el sostenimiento del hogar. Knaul (1993) devela el hurto como actividad laboral realizada por los niños que viven en las calles bogotanas. La explotación laboral de los menores en las zonas rurales (Gutiérrez, 1984; Salazar, 1990) es descrita como una actividad ejercida en el contexto familiar, socialmente aceptada, pero desempeñada en condiciones infrahumanas. Knaul y Castillo (1994) hacen notar que los niños trabajan como mandaderos o mensajeros para sus propias familias. Flórez, Knaul y Méndez (1995) se preguntan por cuántos son y dónde trabajan los niños y los jóvenes colombianos. Respuesta que evidencia la magnitud del problema y las dificultades de cuantificación de la población infantil trabajadora. Flórez y Méndez (1998) hablan de la tendencia decreciente del trabajo infantil y juvenil en Colombia durante los años 1992 a 1996, señalan que uno de cada cincuenta y cinco niños urbanos y uno de cada once niños rurales entre siete a once años, es trabajador. (Jaramillo, 2013, p. 72).

Los gobiernos sucesivos incluyen el tema del trabajo infantil y en general la preocupación por el desarrollo de la infancia en sus respectivos planes de desarrollo. Mejoran el marco institucional y legal para su tratamiento. Pero especialmente en la década de 1990 concurren circunstancias que representan un considerable avance: la nueva Constitución de 1991 hace de los planes de desarrollo compromisos obligatorios del gobernante, en lugar de simples ejercicios retóricos. Acoge en su texto un tratamiento actualizado de la concepción de los niños como ciudadanos y sujetos de derechos. Con ese fundamento y con la asistencia técnica tanto de Unicef como de la OIT, que había creado el Programa Internacional para la Erradicación del Trabajo Infantil (IPEC), se formula el "I Plan de acción para la erradicación del trabajo infantil (1995-1998)".

Se creó el Comité Interinstitucional para la Erradicación del Trabajo Infantil y la Protección del Joven Trabajador, rector en el campo del trabajo infanto-juvenil, encargado de la formulación y el desarrollo de las políticas y los programas conducentes a erradicar la participación de los niños y las niñas en el trabajo y proteger y mejorar las condiciones laborales de los jóvenes. (Álvarez et al., 2003, p. 36). 
En 1999 se suscribió el Convenio 138 de la OIT sobre las "Peores formas del trabajo infantil" y en enero de 2000 se lanzó un "II Plan de acción para la erradicación del trabajo infantil".

Estos planes fueron grandes esfuerzos para generar coordinación interinstitucional y movilizar recursos y acciones de instituciones y en general de la sociedad tanto a nivel nacional como regional. El análisis de los planes hecho por Álvarez et al. (2003) detalla los logros y las limitaciones en la ejecución de los planes. A modo de síntesis de los logros el análisis sugiere:

Algunos avances en la ejecución de los planes nacionales:

- Producción de un número importante de material de sensibilización.

- Visibilización de la problemática de los niños, las niñas y los jóvenes trabajadores para las instituciones y organizaciones.

- Avance en información disponible sobre magnitud y características del trabajo infantil.

- Creación de algunos comités departamentales.

- Capacitación a funcionarios de salud, trabajo, bienestar familiar y gobernaciones.

- Construcción de una mesa de concertación sindical sobre el tema del trabajo infantil.

- Ratificación de los convenios 138 y 182 de la OIT.

- Firma de la Declaración de Cartagena de Indias para la Erradicación del Trabajo Infantil.

- Desarrollo y sistematización de proyectos de intervención. (Álvarez et al., 2003, p. 65).

La movilización institucional y de opinión en torno al problema del trabajo infantil puso en evidencia las deficiencias de la información disponible para hacer una planeación que vaya más allá de metas indicativas. Pese a los diversos y significativos estudios de caso, de alcance local o sectorial las estadísticas generales del orden nacional presentaban vacíos. El DANE, con la cooperación del OIT, mejoró significativamente la información disponible a partir de la Encuesta Nacional de Trabajo Infantil.

En el informe que presenta los resultados de la Encuesta Nacional de Trabajo Infantil del año 2001, el DANE describe así el origen de dicha encuesta:
"Dentro del esfuerzo por obtener de manera sistemática la información requerida para avanzar en la erradicación del trabajo infantil, por su parte la OIT estableció a nivel mundial el Programa de Información Estadística y Monitoreo en Materia de Trabajo Infantil (Simpoc), cuyo objetivo es 'ofrecer a los países un instrumento para obtener y analizar datos, planificar actividades contra el trabajo infantil y evaluar los resultados conseguidos con la ejecución del programa'". El resultado de la cooperación del DANE y el Simpoc, así como de las varias reuniones de especialistas que se habían realizado fue la Encuesta sobre Caracterización de la Población entre 5 y 17 años en Colombia, fue obtener

[...] información necesaria sobre los menores involucrados en actividades económicas y no económicas y las características demográficas y socioeconómicas generales de todos los menores en edad escolar y, para los que trabajan, las condiciones de trabajo, seguridad y aspectos de salud (con énfasis en el tipo, frecuencia y gravedad de las lesiones o enfermedades), así como las razones que tienen para trabajar. La encuesta identificará también las características demográficas y socioeconómicas de los padres de cualquier menor del grupo de 5 a 17 años de edad, puesto que existe correlación entre ellas y la incidencia del trabajo infantil. (DANE y OIT, 2001).

Esta encuesta, conocida bajo la sigla ENTI fue un hito de gran importancia y significó la modernización de los registros estadísticos sobre la infancia, necesidad que había sido advertida insistentemente por investigadores como Salazar (1996, 2000). A partir de esta encuesta se introdujeron módulos sobre trabajo infantil en las encuestas de hogares y se formuló un "III Plan para la erradicación del trabajo infantil" vigente de 2003 a 2006, en 2011 se llevó a cabo una segunda encuesta y en el terreno de los planes nacionales se formuló en 2008 la Estrategia Nacional para Prevenir y Erradicar las Peores Formas de Trabajo Infantil y Proteger al Joven Trabajador 2008-2015.

El III Plan reconoce los avances de los dos anteriores y hace explícito que se basa en las lecciones de estos. Busca incluir el problema del trabajo infantil en los planes de desarrollo territoriales; incorporar 
la erradicación del trabajo infantil en la política para erradicar la pobreza; mejorar el conocimiento de las situaciones especiales en las que trabajan los niños; $y$, en general hacer del espacio municipal el ámbito de la coordinación y de las políticas.

En este III Plan se introdujo como estrategia específica frente al trabajo infantil el programa de transferencias condicionadas "Familias en Acción", para incentivar la asistencia escolar. Consiste en otorgar subsidios condicionándolos a la asistencia a controles de salud o en el caso de los escolares a la asistencia a la escuela.

La evaluación del programa, publicada por la OIT/IPEC (2006) señala que el programa logró un impacto positivo sobre la asistencia escolar pero mucho menor sobre el trabajo infantil, siendo más significativo sobre los niños y niñas de 10 a 13 años, que sobre los jóvenes de 14 a 17. No obstante, el programa incidió sobre la disminución de las horas de trabajo por mes, aunque no sobre el abandono del trabajo. De todas maneras, dada la disminución de las horas trabajadas los investigadores se declaran esperanzados en que, a la larga el programa conducirá al abandono del trabajo y a la erradicación del trabajo infantil.

Adicionalmente la evaluación da cuenta de una enorme cantidad de esfuerzos locales de nivel departamental y municipal, tanto en el campo de la coordinación interinstitucional como de las acciones educativas hacia las familias y, en general las más diversas formas de acción. No cabe duda de que este plan, como los anteriores, logra una movilización institucional y social considerable.

El cuarto plan, es decir la Estrategia Nacional para Prevenir y Erradicar las Peores Formas de Trabajo Infantil y Proteger al Joven Trabajador 20082015, hace honor al hecho de estar precedido por tres planes y más de una década de esfuerzos institucionales. Su diseño institucional tiene en cuenta los aprendizajes de las experiencias anteriores, se centra como lo hizo el tercero en las peores formas de trabajo infantil, concede una enorme importancia a las opciones educativas y a la comprensión de la familia como sujeto de las acciones, se centra en la lucha contra la pobreza extrema. No hemos tenido acceso a una evaluación general de la estrategia cuya fecha final era 2015, pero se puede encontrar documentación sobre la vigencia y permanencia de acciones de instituciones como el ICBF, el Ministerio del Trabajo y muchas alcaldías y gobernaciones, así como organismos internacionales.

En 2015 se cumplieron veinte años de políticas públicas para la erradicación del trabajo infantil que han sido brevemente reseñadas en las líneas anteriores. Nadie podría negar la enorme cantidad de esfuerzos y de recursos empleados en esta tarea, pero a pesar de que se ha reducido, el trabajo infantil sigue allí. Su dimensión en cifras absolutas y su peso porcentual son menores que hace veinte años pero sigue siendo un problema considerable. A continuación, veremos algunas de las tendencias más notorias del trabajo infantil de acuerdo con las estadísticas del DANE de los últimos cinco años.

\section{Tendencias del trabajo infantil entre 2012 y 2016}

Volumen total

El 24 de agosto de 2012 la revista Portafolio publicó una noticia sobre el incremento de los menores trabajadores de acuerdo con las estadísticas del DANE. Decía la nota del diario:

En dos años, cuando la economía ha tenido ritmos notables de crecimiento, alivio en el desempleo y caída en las tasas de pobreza e indigencia, 416.000 menores entre 5 y 17 años llegaron al mundo laboral hasta completar 1.466.000 en el 2011, es decir, creció 39,5 por ciento.

Ese aumento del trabajo infantil se produce a pesar de las campañas para erradicar esa forma de ocupación, y del momento saludable de la economía que llevaría a pensar en una caída de la ocupación laboral de esos niños y adolescentes.

Por el contrario, dicha tasa registró un verdadero disparo, al pasar de 9,2 por ciento en el 2009 a 13 por ciento en el 2011, lo que constituye un motivo de alarma para la sociedad colombiana, según dijo el director del DANE, Jorge Bustamante, al dar a conocer ayer el panorama del trabajo infantil en el país.

Aquí se reseñaba lo afirmado por el director del DANE en la presentación de los resultados de la Encuesta Nacional de Trabajo Infantil (ENTI) 2011, desarrollada por el DANE, el Ministerio del Trabajo 
y el Instituto Colombiano de Bienestar Familiar (ICBF), con el apoyo del Departamento Nacional de Planeación (DNP) y la Organización Internacional del Trabajo (OIT).

En la tabla 1 puede verse la evolución de la tasa de trabajo infantil desde 2001 hasta 2016. La cifra entre los años 2009 y 2011 muestra efectivamente un notable incremento, tal como lo evidenció el DANE y lo reseñó el diario. La alarma expresada era plenamente justificada. No obstante, en los años siguientes, la tasa volvió a un nivel que se podría considerar normal si no fuese un exabrupto considerar normal a una de las realidades más dolorosas del país. El caso es que no siguió aumentando y más bien ha mostrado cierta disminución, todavía muy leve como para ser considerada satisfactoria.

Tabla 1. Tasas de participación en el trabajo infantil, total nacional. Población entre 5 y 17 años.

48

\begin{tabular}{|c|c|c|}
\hline Año & $\begin{array}{c}\text { Tasa trabajo } \\
\text { infantil }\end{array}$ & Número \\
\hline 2001 & 12,9 & 1.409 .777 \\
\hline 2003 & 10,8 & 1.180 .864 \\
\hline 2005 & 8,9 & 999.895 \\
\hline 2007 & 6,9 & 786.567 \\
\hline 2009 & 8,9 & 1.050 .147 \\
\hline 2011 & 13,0 & 1.465 .031 \\
\hline 2012 & 10,2 & 1.160 .000 \\
\hline 2013 & 9,7 & 1.091 .000 \\
\hline 2014 & 9,3 & 1.039 .000 \\
\hline 2015 & 9,1 & 1.018 .000 \\
\hline 2016 & 7,8 & 868.516 \\
\hline
\end{tabular}

Fuente: elaboración propia con base en estadísticas del DANE. GEIH-2016-ENTI 2011.

¿Qué ocurrió entre 2009 y 2011 que pudiese ocasionar este cambio tan notorio? Es difícil, por decir lo menos, aventurar una respuesta concluyente a este interrogante. Pero quizá la respuesta se debe buscar poniendo en duda la afirmación según la cual "la economía ha tenido ritmos notables de crecimiento, alivio en el desempleo y caída en las tasas de pobreza e indigencia"; además la tendencia ascendente se nota ya desde el periodo 2007-2009 y no solamente entre 2009 y 2011. Si se toma como cifra inicial la de 2007, el incremento es mucho mayor de lo que expresa el diario. El Ministerio del Trabajo (2012) analiza los resultados de la encuesta de 2011 y sugiere una respuesta al Ilamativo incremento de la tasa de trabajo infantil:

Entre 2005 y 2009, periodos comparables entre sí, la tasa de trabajo infantil tuvo una variación positiva al pasar del $16 \%$ al $13,6 \%$ en 2007 , pero es revertida en 2009, cuando llega al 15,5\%. Este último impacto está presumiblemente asociado a la crisis económica internacional que incentivó la salida al mercado de trabajo de diferentes miembros de los hogares, incluidos los menores de 18 años. (pp. 4-5).

La crisis económica internacional que menciona el texto corresponde a la recesión que según algunos comprende desde 2008 hasta 2015; se inició en Estados Unidos y sus efectos aún se sienten y la economía mundial no se ha recuperado del todo. Se dice que es la peor crisis económica desde la Gran Depresión de 1929 y hay mucha literatura y discusiones al respecto. En lo que se refiere a Colombia, una publicación del Banco de la República (Arango, Parra y Pinzón, 2015) estudia la relación entre el ciclo económico y el mercado de trabajo. Sus autores afirman que los dos últimos periodos de recesión de la economía ocurrieron entre diciembre de 1997 y junio de 1999, y entre enero de 2008 y marzo de 2009. El efecto de las recesiones se resume por Arango, Parra y Pinzón (2015) de la siguiente manera:

Se encuentra que la participación laboral aumenta en las contracciones mientras que la tasa de ocupación cae. La suma de estos dos hechos permite predecir sin ambigüedades que en las recesiones el desempleo aumenta. En particular, los cesantes, más que los aspirantes, siempre aumentan en los momentos de crisis; es decir, los despidos y la reinserción al mercado parecen ser los eventos más regulares durante los periodos de crisis. Los salarios y las horas trabajadas caen en las recesiones mientras que el subempleo por horas, aumenta. La relación de asalariados a población en edad de trabajar se constituye en una variable clave para el estudio de las fases del ciclo ya que siempre se contrae durante las recesiones. (p. 2). 
Se entiende por participación laboral el hecho de que las personas salgan a buscar trabajo, si no lo encuentran son aspirantes, si se quedan sin empleo, son cesantes. En la crisis aumentan los cesantes y lo hacen también los aspirantes. La medida sugerida por Arango, Parra y Pinzón (2015) de relacionar los asalariados con la población en edad de trabajar como indicador de la recesión incluye, al menos estadísticamente a la parte más significativa del trabajo infantil ya que se considera población en edad de trabajar (PET) a los mayores de 12 años. En este sentido, uno de los efectos previsibles de la crisis es la salida de un gran número de menores al mercado de trabajo. Así pues, la situación señalada en la revista Portafolio (2012) por los directivos del DANE no es tan sorprendente. En presencia de una severa crisis económica de alcance internacional, la dinámica del mercado laboral se comporta como se ha mostrado y esto involucra el trabajo infantil.

Por otra parte, en el extenso periodo ilustrado en la tabla 1, el trabajo infantil ha disminuido en forma notoria en dos momentos, en 2007 antes del inicio de la crisis que se mencionó en las líneas anteriores y en los datos —más recientes de 2016. La continuación de esta tendencia es deseable, pero es imposible pronosticar que se mantendrá. La exclusión social y la pobreza, las secuelas del conflicto armado y grandes males sociales como el tráfico de drogas, la explotación sexual de menores, el uso de niños en la minería ilegal mantienen al 7,8 \% de los niños del país — según las cifras de
2016 - en una condición que no es de ninguna manera admisible.

Conviene señalar aquí que hay una dificultad con los datos, advertida por el mismo DANE: las estadísticas anteriores desde el año 2001 hasta el año 2009 no son comparables con las estadísticas de 2011 en adelante, debido a cambios metodológicos introducidos en el diseño de las muestras y de los instrumentos de investigación. Este problema, que se hace más notorio en cuanto la información esté más desagregada, lleva a algunos analistas a aclarar que no es posible determinar en qué medida los aumentos o disminuciones entre los años 2009 y 2011 se deben a cambios reales o a los cambios metodológicos.

\section{Distribución por áreas geográficas y género}

De acuerdo con el DANE, la población total de Colombia para el año 2016 era de 47'540.354 personas, de las cuales el $77 \%$ estaba en las cabeceras municipales y el restante $23 \%$ en centros poblados menores y rural disperso. Esta clasificación se puede asimilar a las nociones de urbano y rural. La población de Colombia se ha hecho predominantemente urbana. Según esta tendencia, los menores entre 5 y 17 años, niños, niñas y adolescentes (NNADD) son para el mismo 2016 un total de $11^{\prime} 126,198$, esto es el 23,4\% de la población total. En las cabeceras esta proporción disminuye un poco y es del $22,4 \%$, mientras en las zonas rurales la misma proporción asciende al 27,8\%. Esto significa que hay mayor proporción de NNADD en las zonas rurales.

Tabla 2. Trabajo infantil según áreas geográficas y género.

\begin{tabular}{|l|r|r|r|r|r|r|}
\hline & \multicolumn{2}{|c|}{ Total nacional } & \multicolumn{2}{c|}{ Cabeceras } & \multicolumn{2}{c|}{$\begin{array}{c}\text { Centro poblado y rural } \\
\text { disperso }\end{array}$} \\
\hline & Hombres & \multicolumn{1}{c|}{ Mujeres } & Hombres & \multicolumn{1}{c|}{ Mujeres } & \multicolumn{1}{c|}{ Hombres } & Mujeres \\
\hline $\mathbf{2 0 1 2}$ & 795.902 & 363.896 & 394.711 & 243.564 & 401.191 & 120.331 \\
\hline $\mathbf{2 0 1 3}$ & 735.566 & 355.508 & 364.522 & 234.616 & 371.043 & 120.892 \\
\hline $\mathbf{2 0 1 4}$ & 695.180 & 343.996 & 345.941 & 230.967 & 349.239 & 113.029 \\
\hline $\mathbf{2 0 1 5}$ & 694.496 & 323.908 & 337.121 & 211.366 & 357.375 & 112.542 \\
\hline $\mathbf{2 0 1 6}$ & 598.382 & 270.336 & 293.779 & 170.665 & 304.603 & 99.671 \\
\hline
\end{tabular}

Fuente: elaboración propia con base en estadísticas del DANE. GEIH-2015. 
Tabla 3. Trabajo infantil según áreas geográficas y género. Porcentajes.

\begin{tabular}{|l|r|r|r|r|r|r|}
\hline & \multicolumn{2}{|c|}{ Total nacional } & \multicolumn{2}{c|}{ Cabeceras } & \multicolumn{2}{c|}{$\begin{array}{c}\text { Centro poblado y rural } \\
\text { disperso }\end{array}$} \\
\hline & Hombres & \multicolumn{1}{|c|}{ Mujeres } & \multicolumn{1}{c|}{ Hombres } & \multicolumn{1}{c|}{ Mujeres } & \multicolumn{1}{c|}{ Hombres } & \multicolumn{1}{c|}{ Mujeres } \\
\hline $\mathbf{2 0 1 2}$ & 68,6 & 31,4 & 61,8 & 38,2 & 76,9 & 23,1 \\
\hline $\mathbf{2 0 1 3}$ & 67,4 & 32,6 & 60,8 & 39,2 & 75,4 & 24,6 \\
\hline $\mathbf{2 0 1 4}$ & 66,9 & 33,1 & 60 & 40 & 75,5 & 24,5 \\
\hline $\mathbf{2 0 1 5}$ & 68,2 & 31,8 & 61,5 & 38,5 & 76,1 & 23,9 \\
\hline $\mathbf{2 0 1 6}$ & 68,9 & 31,1 & 63,3 & 36,7 & 75,3 & 24,7 \\
\hline
\end{tabular}

Fuente: elaboración propia con base en estadísticas del DANE. GEIH-2016.

En todos los años observados, los hombres se vinculan al mercado de trabajo mucho más que las mujeres. La proporción en el total nacional es cercana a 70 hombres por 30 mujeres de cada 100 NNAD que trabajan. Si se desagregan las cabeceras, o sea las zonas urbanas, crece la proporción de mujeres, aunque los hombres siguen siendo la mayoría. En las zonas rurales, por el contrario, vuelve a ser predominante la vinculación masculina al trabajo y la proporción del trabajo infantil femenino se reduce.

En consonancia con la tendencia nacional, en números absolutos la población de NNAD trabajadores disminuye, pero las proporciones porcentuales se conservan casi iguales en los cinco años observados.

\section{Niños y adolescentes}

La franja de edad entre los 5 y 17 años se divide en dos segmentos para fines estadísticos y legales: de 5 a 14 años y de 15 a 17. Los primeros serían niños y niñas, y los segundos adolescentes. La ley establece la posibilidad de que las autoridades otorguen autorización para trabajar a los adolescentes a partir de los 15 años. Es claro que la mayor capacidad de trabajo por su desarrollo y la mayor autonomía los hace más susceptibles de vinculación laboral.

Entre 2012 y 2016 la población total de 5 a 14 años pasó de 8.671.652 a 8'536.079, y la población de 15 a 17, pasó de 2'660.284 a 2'590.119. Esta tendencia a la paulatina y lenta disminución corresponde a la llamada transición demográfica. El país está empezando a transitar por el aumento tanto relativo como absoluto de los grupos de edad mayor y la disminución también en términos absolutos y en peso relativo de los grupos jóvenes; sin embargo, en el mediano plazo los niños y jóvenes seguirán siendo el segmento más grande de la población del país. En el primer grupo, de 5 a 14 años, la tasa de trabajo (TTI) es 4,6\%, mientras que en el segundo grupo se eleva a $24,4 \%$. Lo que significa que de cada cuatro adolescentes colombianos uno trabaja. Esta cifra deja ver con mucha claridad la gravedad de la situación.

Tabla 4. Tasas de trabajo infantil por grupos de edad y áreas geográficas en 2012 y 2016.

\begin{tabular}{|c|c|c|c|c|c|c|}
\hline & \multicolumn{2}{|c|}{ Total } & \multicolumn{2}{|c|}{ Cabeceras } & \multicolumn{2}{|c|}{ Centro poblado y rural } \\
\hline & 2012 & 2016 & 2012 & 2016 & 2012 & 2016 \\
\hline 5 a 14 años & 5,9 & 4,2 & 4,0 & 2,5 & 11 & 8,7 \\
\hline 15 a 17 años & 24,3 & 19,8 & 19,6 & 15,9 & 37,6 & 31,7 \\
\hline
\end{tabular}

Fuente: elaboración propia con base en estadísticas del DANE. GEIH-2016. 
La tendencia a la disminución en las tasas de trabajo (TTI) afecta por igual los dos grupos de edad y las áreas geográficas.

El principal problema de la vinculación temprana al trabajo es su impacto sobre las oportunidades de educación por parte de los NNAD, es decir sobre su proceso de formación de capital humano con las consecuencias que ello tiene para sus oportunidades futuras, ya sea porque los aleja y excluye de la educación, o porque les dificulta el rendimiento adecuado. En el total de la población de 5 a 17 años la asistencia escolar es de $90,3 \%$, un poco más alta en las zonas urbanas $(91,7 \%)$ y algo más reducida en las zonas rurales $(86,4 \%)$. Pero en la población de NNAD trabajadores la escolaridad se reduce a $67,2 \%$ de asistencia escolar en el total nacional, que corresponde a $70,6 \%$ en las zonas urbanas y $63,3 \%$ en las rurales.

La falta de oportunidades de estudio puede deberse a muchos factores, no se refiere simplemente a que los jóvenes se vean obligados a trabajar y deban abandonar el estudio. Podemos mencionar, entre otros, la distancia entre el lugar de residencia y la escuela, las dificultades para costear el transporte, la falta de cupos escolares, el bajo rendimiento académico de los NNAD trabajadores, forman parte de una gama de condiciones y carencias tanto personales como familiares e institucionales que se convierten en expulsores de la educación. Estas situaciones afectan especialmente a los adolescentes que se encuentran en los años finales de su ciclo de formación, ya que en cuanto a la educación primaria y básica secundaria las coberturas son altas. Se espera que a los 15 años un joven esté concluyendo la educación básica (noveno grado), pero a partir de este punto, la educación media, los últimos años para obtener el grado de bachiller, disminuye significativamente en cuanto a su cobertura. De acuerdo con un documento oficial del año 2014:

En 2012 la TCN (tasa de cobertura neta) en las áreas urbanas para el nivel de educación secundaria fue de $78,9 \%$ y en la zona rural de 54,8 \%. En el nivel de media las tasas para las zonas urbana y rural fueron 47,8 y $24,9 \%$, respectivamente. En ambos niveles, la diferencia entre las tasas de la zona urbana y rural es superior a 20 puntos porcentuales. (Conpes y DNP, 2014, p. 18).
El adolescente trabajador, en estas circunstancias, forma parte de un todo aún más complejo, que no se suele percibir desde el punto de vista de los estudios sobre trabajo infantil, que tienden a mirar el problema de forma un tanto unilateral, como actividades socialmente indeseables, que es preciso desestimular e incluso reprimir. Si bien este enfoque puede tener sentido en el caso de los niños y niñas con edades menores a los 14 años, los adolescentes están entrando en una situación que se caracteriza por la disminución de sus oportunidades de desarrollo personal, que podrían ganarles un mejor lugar en el mercado laboral y sobre ellos crece la presión para la vinculación temprana al trabajo.

Las condiciones de trabajo de los NNAD

La OIT ha introducido el concepto de trabajo decente para enfatizar que hay un derecho humano fundamental al trabajo, no de cualquier clase, sino bajo condiciones específicas de dignidad.

Un trabajo productivo con remuneración justa, seguridad en el lugar de trabajo y protección social para el trabajador y su familia, mejores perspectivas para el desarrollo personal y social, libertad para que manifiesten sus preocupaciones, se organicen y participen en la toma de decisiones que afectan a sus vidas así como la igualdad de oportunidades y de trato para mujeres y hombres. (2007, citado por Zorro y Patiño, 2013, p. 119).

El trabajo decente constituye un objetivo que puede fundamentar la política social y laboral desde una perspectiva de desarrollo humano, de manera más integral que la eliminación del trabajo infantil, la ampliación de la cobertura educativa o la generación de puestos de trabajo, por cuanto integra a todas ellas.

Este concepto, aunque es válido como principio para toda la población y como fundamento ideal de la política laboral, es especialmente importante para los jóvenes que están iniciando su ingreso al mundo laboral y cuya trayectoria dependerá, en la mayoría de los casos, de las condiciones de su inserción inicial. ¿Han hecho esa inserción inicial en condición de NNAD trabajadores? ¿Significa eso 
una ventaja o lo contrario? Hace falta mayor conocimiento y precisión estadística, pero se puede afirmar sin temor a equivocación, que el ingreso al trabajo en condiciones de trabajo infantil conlleva una alta probabilidad de prolongar las condiciones de pobreza y exclusión.

Las posiciones ocupacionales de la población de NNAD trabajadores para 2016 se muestran en la tabla 5 .

El $45 \%$ en el total nacional y el $57 \%$ en las zonas rurales trabajan sin remuneración. Los independientes, por su parte, en esta población son con plena certeza oficios informales y los asalariados lo son también en una proporción muy grande. Nada más lejano de la noción de trabajo decente.

\section{Remuneración}

Un indicador adicional de la informalidad son los ingresos laborales, como se puede ver en la tabla 6 .

Todos están por debajo de un salario mínimo, lo cual es un indicador de informalidad. En estas 52 condiciones es improbable que haya protección y seguridad social. Si se suman los tres rangos de remuneración más baja, es decir desde medio salario mínimo hasta ninguna remuneración, tendremos el $82 \%$ en el total nacional y el $89 \%$ en las zonas rurales. En las cabeceras la situación no es mucho mejor y llega al $76 \%$.

\section{Jornadas de trabajo}

En relación con los ingresos están las jornadas de trabajo. El número de horas semanales trabajadas se puede ver en la tabla 7 para el año 2016.

Las jornadas largas, de más de 30 horas, son frecuentes $(36 \%)$, pero predominan las jornadas breves, de menos de 15 horas. El panorama conjunto que nos muestran estos datos es el de trabajos de baja productividad, muy mal remunerados y en condiciones de informalidad. En resumen, el trabajo infantil en la forma en que se da en Colombia, además de ser en sí mismo una negación de los derechos de los NNAD, es completamente opuesto a la idea de un trabajo decente o digno.

Tabla 5. Población de 5 a 17 años que trabaja según posición ocupacional 2016.

\begin{tabular}{|l|r|r|r|r|r|r|}
\hline Concepto & Total nacional & \multicolumn{1}{c|}{ \% } & \multicolumn{1}{c|}{ Cabeceras } & & \multicolumn{2}{|c|}{ Rural } \\
\hline Población de 5 a 17 años que trabaja & 1.018 .404 & 100 & 548.487 & 100 & 469.918 & 100 \\
\hline Asalariados & 291.172 & 29 & 197.258 & 36 & 93.915 & 20 \\
\hline Independientes & 273.167 & 27 & 164.190 & 30 & 108.977 & 23 \\
\hline Trabajadores sin remuneración & 454.065 & 45 & 187.040 & 34 & 267.025 & 57 \\
\hline
\end{tabular}

Fuente: elaboración propia con base en estadísticas del DANE. GEIH-2016.

Tabla 6. Población de 5 a 17 años que trabaja según rangos de ingresos laborales recibidos el mes pasado.

\begin{tabular}{|l|r|r|r|r|r|r|}
\hline Concepto & Total nacional & \multicolumn{1}{c|}{ \% } & \multicolumn{1}{c|}{ Cabeceras } & \multicolumn{1}{c|}{ Rural } & \% \\
\hline Población de $\mathbf{5}$ a $\mathbf{1 7}$ años que trabaja & 1.018 .404 & 100 & 548.487 & 100 & 469.918 & 100 \\
\hline Sin remuneración o ganancia & 479.194 & 47 & 202.948 & 37 & 276.245 & 59 \\
\hline Hasta 1/4 SMMLV & 223.277 & 22 & 144.740 & 26 & 78.537 & 17 \\
\hline Entre 1/4 y 1/2 SMMLV & 131.765 & 13 & 72.442 & 13 & 59.323 & 13 \\
\hline Más de 1/2 SMMLV & 168.378 & 17 & 117.075 & 21 & 51.303 & 11 \\
\hline No informa & 15.790 & 2 & 11.282 & 2 & 4.508 & 1 \\
\hline
\end{tabular}

Fuente: elaboración propia con base en estadísticas del DANE. GEIH-2016. 


\section{Razones para trabajar}

¿Por qué trabajan los NNAD? La información obtenida por el DANE sobre trabajo infantil incluye las razones que los sujetos aducen para trabajar. La tabla 8 presenta los resultados para el año 2015.

Las dos razones principales son: ayudar a la familia y tener su propio dinero, en todos los años de 2012 a 2015 se conserva esta tendencia; sin embargo, Ilama la atención que en las zonas urbanas tiende a predominar el tener dinero propio, factor de autonomía y empoderamiento del NNAD frente al núcleo familiar, mientras en las zonas rurales predomina la responsabilidad económica hacia la familia.

\section{El trabajo doméstico}

La medición del trabajo infantil en las Encuestas de Hogares emplea dos medidas complementarias: la primera es el trabajo u ocupación propiamente, que puede ser o no remunerado y que muestra la vinculación de los NNAD al mercado laboral, esta medida corresponde a los análisis tradicionales sobre temas laborales. Se ha introducido una segunda medida que tiene que ver con el uso del tiempo y que permite determinar si los NNAD hacen oficios domésticos en el propio hogar y cuánto tiempo dedican a ello. Si dedican 15 horas o más a la semana, al trabajo doméstico, que por definición no es remunerado, se incluyen en una segunda medida de trabajo ampliado. Se considera que esta dedicación a los oficios del hogar constituye una carga que puede tener graves efectos sobre Ios NNAD al obstaculizar o sustituir el estudio. La comparación entre estas dos formas de actividad ofrece una perspectiva más ajustada a lo que significa el trabajo de los menores y en particular ofrece una interesante aproximación a las diferencias por género.

Tabla 7. Población de 5 a 17 años que trabaja según horas semanales.

\begin{tabular}{|l|r|r|r|r|r|r|}
\hline Concepto & Total nacional & & \multicolumn{1}{c|}{ Cabeceras } & & \multicolumn{1}{l|}{ Rural } & \\
\hline Población de 5 a 17 años que trabaja & 1.018 .404 & 100 & 548.487 & 100 & 469.918 & 100 \\
\hline Menos de 15 horas & 396.172 & 39 & 216.238 & 39 & 179.932 & 38 \\
\hline De 15 a 29 horas & 254.989 & 25 & 131.034 & 24 & 123.955 & 26 \\
\hline 30 horas y más & 366.659 & 36 & 200.628 & 37 & 166.029 & 35 \\
\hline No informa & 586 & 0 & 586 & 0 & - \\
\hline
\end{tabular}

Fuente: elaboración propia con base en estadísticas del DANE. GEIH-2015.

Tabla 8. Población de 5 a 17 años que trabaja según razones por las que trabaja.

\begin{tabular}{|c|c|c|c|c|c|c|}
\hline Concepto & Total nacional & & Cabeceras & & Rural & \\
\hline Población de 5 a 17 años que trabaja & 1.018 .404 & 100,0 & 548.487 & 100,0 & 469.918 & 100,0 \\
\hline $\begin{array}{l}\text { Debe ayudar con los gastos de la casa, } \\
\text { ayudar a costearse el estudio }\end{array}$ & 111.453 & 10,9 & 56.810 & 10,4 & 54.643 & 11,6 \\
\hline $\begin{array}{l}\text { Porque el trabajo lo forma, lo hace honrado } \\
\text { y lo aleja de los vicios }\end{array}$ & 126.264 & 12,4 & 55.625 & 10,1 & 70.640 & 15,0 \\
\hline $\begin{array}{l}\text { Debe participar en la actividad económica } \\
\text { de la familia }\end{array}$ & 346.993 & 34,1 & 143.993 & 26,3 & 203.001 & 43,2 \\
\hline Le gusta trabajar para tener su propio dinero & 393.544 & 38,6 & 264.699 & 48,3 & 128.845 & 27,4 \\
\hline Otra razón & 40.150 & 3,9 & 27.360 & 5,0 & 12.790 & 2,7 \\
\hline
\end{tabular}

Fuente: elaboración propia con base en estadísticas del DANE. GEIH-2015. 
De acuerdo con la tabla 9, el total de NNAD bajo el concepto de trabajo infantil ampliado es de 1.395.777 (Reátiga, 2013, p. 14) ${ }^{3}$.

Como se puede apreciar en las tablas 9 y 10, el número de hombres trabajando prácticamente duplica al número de mujeres. Pero cuando se trata de los oficios del hogar el número de mujeres es considerablemente mayor que el de los hombres. La tendencia se mantiene con poca variación a lo largo de los cinco años reseñados. Difícilmente se puede atribuir a la pobreza extrema la dedicación de las niñas a este tipo de tareas. Lo que parece haber aquí es más bien una pauta cultural. Por otra parte, en cuanto se refiere al trabajo remunerado, son los hombres quienes con más frecuencia se vinculan a él, aunque también lo hace un buen número de mujeres. En este caso es mucho más probable que sea la pobreza y la necesidad de ingresos adicionales la explicación de la vinculación al trabajo de unos y otras.

El tema del trabajo y los oficios del hogar según género ofrece una mirada interesante si se analiza desde lo urbano-rural. En esta distribución geográfica según sexos, aparecen rasgos que revelan aspectos tanto del mercado laboral como de las pautas culturales que rodean el trabajo de los NNAD.

La situación cambia cuando se trata de la realización de oficios del hogar.

Dentro de la tendencia a que los oficios del hogar recaigan de preferencia sobre las niñas, puede verse que en las zonas rurales esta proporción es aún mayor. El número de NNAD que hace oficios en Colombia es de 6'640.461, distribuidos en 3'153.583 (47,5\%) hombres y 3'486.878 (52,5\%) mujeres. ¿Qué clase de oficios llevan a cabo según género?

Tabla 9. Trabajo y oficios en el hogar por género. Población de 5 a 17 años. Total nacional.

\begin{tabular}{|c|c|c|c|c|c|c|c|}
\hline \multirow{2}{*}{ Año } & \multicolumn{4}{|c|}{ Trabaja } & \multicolumn{2}{c|}{ Realiza oficios del hogar por 15 horas o más } & \multirow{2}{*}{ Total } \\
\cline { 2 - 8 } & Hombres & Mujeres & Total & Hombres & Mujeres & Total & \\
\hline $\mathbf{2 0 1 2}$ & 795.902 & 363.896 & 1.159 .798 & 149.795 & 484.986 & 634.781 & $1^{\prime} 794.579$ \\
\hline $\mathbf{2 0 1 3}$ & 735.766 & 355.508 & 1.091 .274 & 120.909 & 458.089 & 578.998 & $1^{\prime} 670.272$ \\
\hline $\mathbf{2 0 1 4}$ & 695.180 & 343.996 & 1.039 .176 & 99.604 & 412.788 & 512.392 & $1^{\prime} 551.568$ \\
\hline $\mathbf{2 0 1 5}$ & 694.496 & 323.908 & 1.018 .404 & 118.127 & 429.027 & 547.154 & $1^{\prime} 565.558$ \\
\hline $\mathbf{2 0 1 6}$ & 598.382 & 270.336 & 868.718 & 116.988 & 410.071 & 527.059 & $1^{\prime} 395.777$ \\
\hline
\end{tabular}

Fuente: elaboración propia con base en estadísticas del DANE. GEIH-2016.

Tabla 10. Trabajo y oficios en el hogar según género. Relación porcentual.

\begin{tabular}{|c|c|c|c|c|c|c|}
\hline \multirow{2}{*}{ Año } & \multicolumn{3}{|c|}{ Trabaja } & \multicolumn{3}{c|}{ Realiza oficios del hogar por 15 horas o más } \\
\cline { 2 - 7 } & Hombres & Mujeres & Total & Hombres & Mujeres & Total \\
\hline $\mathbf{2 0 1 2}$ & 69 & 31 & 100 & 24 & 76 & 100 \\
\hline $\mathbf{2 0 1 3}$ & 67 & 33 & 100 & 21 & 79 & 100 \\
\hline $\mathbf{2 0 1 4}$ & 67 & 33 & 100 & 19 & 71 & 100 \\
\hline $\mathbf{2 0 1 5}$ & 68 & 32 & 100 & 22 & 78 & 100 \\
\hline $\mathbf{2 0 1 6}$ & 69 & 31 & 100 & 22 & & 100 \\
\hline
\end{tabular}

Fuente: elaboración propia con base en estadísticas del DANE. GEIH-2016.

\footnotetext{
3 Reátiga menciona que en 2002 una publicación de Unicef, utilizando datos de 1996 decía: “En Colombia trabajan más de 2’500.000 niños y niñas. De este total, $1^{\prime} 700.000$ son adolescentes entre 12 y 17 años y 800.000 son niños y niñas de entre 6 y 11 años. El $80 \%$ trabaja en el sector informal. 323.000 niños y niñas se encuentran trabajando en el servicio doméstico en hogares de terceros". La cifra de Unicef no incluye los que trabajan en el propio hogar que con seguridad elevarían el total por encima de los 3'500.000. Las cosas han cambiado en veinte años, la población ha crecido y el número de infantes trabajadores ha disminuido. Sin embargo, aún estamos muy lejos de algo que se pueda denominar erradicación.
} 
Tabla 11. Oficios del hogar por 15 horas o más.

\begin{tabular}{|r|r|r|r|r|r|r|}
\hline & \multicolumn{2}{|c|}{ Total nacional } & \multicolumn{2}{c|}{ Cabeceras } & \multicolumn{2}{c|}{ Centro poblado y rural disperso } \\
\hline & Hombres & \multicolumn{1}{c|}{ Mujeres } & \multicolumn{1}{c|}{ Hombres } & \multicolumn{1}{c|}{ Mujeres } & \multicolumn{1}{c|}{ Hombres } & \multicolumn{1}{c|}{ Mujeres } \\
\hline $\mathbf{2 0 1 2}$ & 149.795 & 484.986 & 107.769 & 304.570 & 42,025 & 180,415 \\
\hline $\mathbf{2 0 1 3}$ & 120.909 & 458.089 & 85.433 & 267.755 & 35,476 & 190,334 \\
\hline $\mathbf{2 0 1 4}$ & 99.604 & 412.788 & 70.729 & 251.683 & 28,875 & 161,105 \\
\hline $\mathbf{2 0 1 5}$ & 118.127 & 429.027 & 75.024 & 269.571 & 43,103 & 159,456 \\
\hline $\mathbf{2 0 1 6}$ & 116.988 & 410.071 & 81.815 & 260.868 & 35.173 & 149.203 \\
\hline
\end{tabular}

Fuente: elaboración propia con base en estadísticas del DANE. GEIH-2015.

Tabla 12. Oficios del hogar por 15 horas o más. Porcentajes.

\begin{tabular}{|c|c|c|c|c|c|c|}
\hline & \multicolumn{2}{|c|}{ Total nacional } & \multicolumn{2}{c|}{ Cabeceras } & \multicolumn{2}{c|}{ Centro poblado y rural disperso } \\
\hline & Hombres & Mujeres & Hombres & Mujeres & Hombres & Mujeres \\
\hline $\mathbf{2 0 1 2}$ & 23,6 & 76,4 & 26,1 & 73,9 & 18,9 & 81,1 \\
\hline $\mathbf{2 0 1 3}$ & 20,9 & 79,1 & 24,2 & 75,8 & 15,7 & 84,3 \\
\hline $\mathbf{2 0 1 4}$ & 19,4 & 80,6 & 21,9 & 78,1 & 15,2 & 84,8 \\
\hline $\mathbf{2 0 1 5}$ & 21,6 & 78,4 & 21,8 & 78,2 & 21,3 & 78,7 \\
\hline $\mathbf{2 0 1 6}$ & 22,2 & 77,8 & 23,9 & 76,1 & 19,1 & 80,9 \\
\hline
\end{tabular}

Fuente: elaboración propia con base en estadísticas del DANE. GEIH-2015.

Tabla 13. Población de 5 a 17 años que realiza oficios en el hogar.

\begin{tabular}{|l|r|r|r|}
\hline \multicolumn{1}{|c|}{ Concepto } & Total & Hombres & \multicolumn{1}{c|}{ Mujeres } \\
\hline Lavar & 29,8 & 20,9 & 38,0 \\
\cline { 2 - 4 } Planchar & 3,5 & 2,2 & 4,7 \\
\cline { 2 - 4 } Cocinar & 21,8 & 13,2 & 29,7 \\
\cline { 2 - 4 } Cuidar niños pequeños o personas enfermas o con discapacidades, del hogar & 12,4 & 9,2 & 15,3 \\
\cline { 2 - 4 } Atender la huerta casera, cría y cuidado de animales & 9,5 & 10,4 & 8,6 \\
\cline { 2 - 4 } Hacer mandados o mercados & 78,6 & 83,0 & 74,6 \\
\cline { 2 - 3 } Limpieza y mantenimiento del hogar & 83,9 & 78,8 & 88,6 \\
\cline { 2 - 4 } & 2,4 & 2,5 & 2,3 \\
\hline
\end{tabular}

Fuente: elaboración propia con base en estadísticas del DANE. GEIH-2015.

La diferencia de los trabajos realizados según género radica en que en la ciudad ellos hacen mandados o compras y en el campo atienden la huerta casera, la cría y el cuidado de animales; en mínima medida cocinan o cuidan de otros miembros del hogar; mientras que ellas se dedican a la limpieza y al mantenimiento de sus casas, a cuidar niños pequeños o enfermos, a cocinar, lavar y planchar ropa. En las zonas rurales el cuidado de los animales y las labores agrícolas son mayoritariamente ejercidas por los hombres, aunque no excluyentes para las mujeres quienes en el mundo rural atienden simultáneamente y en amplias jornadas de trabajo tanto los quehaceres domésticos como las labores agrícolas (Jaramillo, 2013, p. 74). 
No es difícil ver plasmados en esta distribución los roles tradicionales de género. Tampoco podemos perder de vista que cualquiera que sea el oficio, la dedicación de más de 15 horas semanales es una dura carga para los NNAD y un serio obstáculo a su escolaridad.

\section{¿Por qué no se logra erradicar el traba- jo infantil?}

Es muy difícil responder a esta pregunta. El caso es que pese a la magnitud de los esfuerzos desplegados en intervenciones de variada índole, el trabajo infantil sigue teniendo un nivel muy alto. Las intervenciones institucionales tienen usualmente un enfoque de restitución de derechos, trabajan con NNA que están en el trabajo y les ofrecen educación, con diversas metodologías, algunas muy exitosas. Por otra parte, y en una acción preventiva los programas sociales orientados a las familias focalizan la extrema pobreza y mediante subsidios alivian su situación y les proporcionan servicios. Todo esto está muy bien. Pero el interrogante sigue vigente.

El trabajo infantil, como bien lo señalan todos los análisis sobre el tema, es, en una proporción grande, una estrategia de supervivencia por parte de las familias. Los programas sociales orientados a las familias en extrema pobreza tienen en cuenta este factor, pero, como señala un investigador, existen "familias que no cumplen con los criterios para considerarlas beneficiarias de los subsidios condicionados y que, paradójicamente, deben continuar luchando por alcanzar un ingreso básico de subsistencia con la fuerza laboral de sus hijos e hijas" (Fábregas, 2015, 149-150).

Una parte muy considerable de la creación de empleos en Colombia corresponde a empleo informal. La OIT, con base en la Encuesta de Hogares, señala que en 2012 el $56 \%$ del empleo era informal no agrícola (OIT y Forlac, 2014). Aunque el contexto es un informe sobre los progresos de la formalización, lo que llama la atención es que más de la mitad del empleo es informal. Como se señaló antes, el empleo juvenil, de acuerdo con las estadísticas es predominantemente informal.

A lo anterior se debe añadir que las reformas laborales de comienzos de la década de 2000, con el propósito de disminuir los costos laborales en el sentido de abaratar los costos de despido e introducir formas de contratación sin costos laborales por la vía de la subcontratación, impulsaron la precarización del trabajo: empleos de baja calidad y corta duración; baja estabilidad laboral y desconocimiento de los derechos de los trabajadores. Esta situación pone a las familias en condiciones de vulnerabilidad que no necesariamente se corresponden con la pobreza extrema.

Como bien señala Fábregas (2015):

El alcance del déficit del trabajo decente en Colombia se refleja en la ausencia de los derechos sociales básicos de la fuerza laboral como producto de las reformas laborales; de un adecuado funcionamiento de los mercados laborales depende el hecho de que una gran parte de esta población pueda gozar de tales derechos. Por razones como esta es que la promulgación de políticas dirigidas a la generación de trabajo decente representa la esencia de la política social. (p. 160).

Quizá la dificultad más grande estriba en que gran parte de la prevención del trabajo infantil no tiene que ver directamente con políticas de trabajo infantil sino con la creación de un entorno protector para los niños, los jóvenes y las familias, que logre detener o aminorar el flujo constante de niños hacia el trabajo. Es posible que garantizar trabajo estable y digno, justamente remunerado para las personas cabeza de familia, tenga un impacto sobre la disminución del trabajo infantil mayor que las acciones directas. En condiciones donde abundan los hogares cuya cabeza de familia es una mujer, las políticas para garantizar la igualdad de oportunidades, de remuneración y remover la discriminación de género pueden tener un efecto similar. En palabras de Fábregas (2015), la cuestión no es tanto de pobreza como de desigualdad. Este último es un concepto que tiene que ver con oportunidades en diversos ámbitos de la vida social. En su remoción puede estar la clave de mayores logros en la erradicación del trabajo infantil.

\section{Referencias}

Aldana, E. (1983). Trabajo de los niños matriculados en las escuelas públicas. Mimeo. Instituto Ser. 
Álvarez, L.; Durán, E.; Torrado, M. C.; Vargas, E.; Wilches, R. (2003). Análisis de la política nacional frente al trabajo infantil en Colombia 1995-2002. Serie: Documento de Trabajo, 159. Bogotá: Observatorio Sobre Infancia de la Universidad Nacional de Colombia, OIT/IPEC.

Arango, L. E.; Parra, F. F.; Pinzón, A. J. (2015). El ciclo económico y el mercado de trabajo en Colombia: 1984-2014. Borradores de Economía, 911.

Ayala, U.; Becerra, C. (1982). El trabajo infantil en Bogotá. Bogotá: Centro de Estudios sobre Desarrollo Económico (CEDE), Facultad de Economía, Universidad de los Andes.

Consejo Nacional Política Económica y Social (Conpes), Departamento Nacional de Planeación (DNP). (3 de julio de 2014). Documento Conpes 173. Lineamientos para la generación de oportunidades para los jóvenes. Bogotá.

Departamento Administrativo Nacional de Estadística (DANE). (2011). Encuesta de calidad de vida 2011. Recuperado de: http://www.dane. gov.co/files/investigaciones/condiciones vida/ calidad vida/ECV 2011 Bogota.pdf

Departamento Administrativo Nacional de Estadística (DANE). (2013). Boletín de prensa n. ${ }^{12}$. Bogotá.

Departamento Administrativo Nacional de Estadística (DANE). (2015). Trabajo infantil. Principales resultados. Bogotá.

Departamento Administrativo Nacional de Estadística (DANE). (13 de abril de 2016). Boletín Técnico. Trabajo Infantil. Octubre-diciembre 2015. Bogotá.

Departamento Administrativo Nacional de Estadística (DANE), Organización Internacional del Trabajo (OIT). (2001). Análisis de los resultados de la encuesta sobre caracterización de la población entre 5 y 17 años en Colombia. Encuesta Nacional de Trabajo Infantil. Bogotá.

Fábregas, J. J. (2015). Consideraciones para la optimización de la Estrategia nacional de prevención y erradicación de las peores formas de Trabajo Infantil y protección al Joven Trabajador 2008-2015. Papel Político, 20(1): 147-178. Recuperado de: http://dx.doi.org/10.11144//averiana.papo20-1.coen

Flórez, C. E.; Knaul, F.; Méndez, R. (1995). Niños y jóvenes: ¿Cuántos y dónde trabajan? Bogotá:
Ministerio de Trabajo y Seguridad Social, CEDE, Facultad de Economía, Universidad de los Andes. Flórez, C. E.; Méndez, R. (1998). Niñas, niños y jóvenes trabajadores. Colombia 1996. OIT. Bogotá: Tercer Mundo.

Jaramillo, M. P. (2013). El trabajo infantil. El caso de "El Prado" en Bogotá, D.C. (Colombia). Tesis doctoral. Salamanca: Universidad de Salamanca.

Knaul, F.; Castillo, Z. (1994). Análisis de situación de menores en circunstancias especialmente difíciles y en alto riesgo en Colombia. Informe final. Unicef Colombia.

Ministerio del Trabajo. (2012). Informe sobre el comportamiento del trabajo infantil en Colombia según la ENTI 2011. Bogotá (Colombia).

Muñoz, C.; Palacios, M. (1978). Aportes al estudio del trabajo infantil en Colombia: Encuesta sobre los niños trabajadores de la clase baja en Bogotá. Bogotá.

Organización de las Naciones Unidas (ONU). (1959). Declaración de los Derechos del Niño. Recuperado de: http://www.humanium.org/ es/declaracion-de-los-derechos-del-nino-texto-completo/

Organización Internacional del Trabajo (OIT). (s.f.). ¿Qué se entiende por trabajo infantil? Recuperado de: http://www.ilo.org/ipec/facts/lang--es/ index.htm

Organización Internacional del Trabajo (OIT). (1999). Las peores formas de trabajo infantil. Convenio n. ${ }^{\circ} 182$ de la Conferencia: 8.7

Organización Internacional del Trabajo (OIT) - Programa Internacional para la Erradicación del Trabajo Infantil (IPEC) - Comité Interinstitucional para la Erradicación del Trabajo Infantil y la Protección de los Jóvenes Trabajadores. (2003). III Plan Nacional para la Erradicación del Trabajo Infantil y la Protección del Trabajo Juvenil 2003-2006. Bogotá.

Organización Internacional del Trabajo (OIT) - Programa Internacional para la Erradicación del Trabajo Infanti (IPEC) - Econometría. (2006). Valoración de los avances del III Plan Nacional de Erradicación del Trabajo Infantil y Protección del Trabajo Juvenil, y recomendaciones para el IV Plan. Bogotá: Oficina Internacional del Trabajo. 
Organización Internacional del Trabajo (OIT) y Programa de Promoción de la Formalización en América Latina y el Caribe (Forlac). (2014). Evolución del empleo informal en Colombia: 2009-2013. Recuperado de: http://www.ilo. org/wcmsp5/groups/public/---americas/---ro-lima/documents/publication/wcms 245615.pdf

Redacción de Economía y Negocios. (24 de agosto de 2012). Mitad de los niños que trabajan lo hacen de manera gratuita. Portafolio.

Reátiga, M. E. (2013). Ser adolescente hoy en Colombia. Barranquilla: Universidad del Norte Editorial.

Salazar, M. C. (1990). Niños y jóvenes trabajadores: Buscando un futuro mejor. Bogotá: Universidad Nacional de Colombia.

Salazar, M. C. (1996). El trabajo Infantil en América Latina. Revista Colombiana de Educación, 33. Recuperado de: http://revistas.pedagogica.edu. co/index.php/RCE/article/view/5397/4424

Salazar, M. C. (2000). El trabajo infantil en Colombia: tendencias y nuevas políticas. Nómadas, 12: 152-159.

Salazar, M. C. (s.f.). La significación social del trabajo infantil y juvenil en América Latina y El Caribe. Ponencia presentada en el 48 Congreso Internacional de Americanistas, Estocolmo (Suecia), 4-9 de julio de 1994. Universidad Pedagógica Nacional.

Zorro, C.; Patiño, I. (2013). Trabajo decente para los jóvenes en Bogotá: contexto, realidades y perspectivas. En: J. Pineda (comp.) (2013). El trabajo decente en Bogotá: diagnóstico y perspectivas (pp. 115-158). Bogotá: Universidad de los Andes, Centro Interdisciplinario de Estudios sobre Desarrollo (Cider), Secretaría Distrital de Desarrollo Económico.

\section{Bibliografía complementaria ${ }^{4}$}

Amar, J. y Madariaga, C. (2009). Intervención psicosocial para la erradicación y prevención del trabajo infantil. Barranquilla: Uninorte.

\footnotetext{
${ }^{4}$ Aunque los textos referenciados no se citan en el presente artículo, contienen conceptos, análisis y opiniones que fundamentaron el contenido y son pertinentes para profundizar en la materia. (Nota de la autora).
}

Departamento Administrativo Nacional de Estadística (DANE). (s.f.). Análisis en profundidad y términos comparativos de los años 2001, 2003, 2005 y 2007 sobre trabajo infantil. Bogotá.

Departamento Administrativo Nacional de Estadística (DANE) y Organización Internacional del Trabajo (OIT). (2011). Trabajo Infantil en Colombia. Bogotá.

Durán, E.; Torrado, M. C. (2007). Derechos de los niños y las niñas. Debates realidades y perspectivas. Bogotá: Universidad Nacional de Colombia.

Grootaert, Ch.; Kanbur, R. (1994). Perspectiva económica del trabajo infantil. Revista Internacional del Trabajo, 114(2): 211-229.

Khoudour-Castéras, D. (2009) Efectos de la migración sobre el trabajo infantil en Colombia. Revista de Economía Institucional, 11(20): 229-252.

Leyra, B. (2009). Aproximaciones antropológicas a la infancia trabajadora: deconstruyendo los mitos y analizando los vacíos de una compleja relación. En: M. I. Jociles, A. Francé y D. Poveda (eds.). Etnografías de la infancia: discursos, prácticas y campos de acción. Madrid: Libros de la Catarata.

Ministerio de Salud y Seguridad Social. (2010). El trabajo infantil en Colombia. Reporte de resultados del Sistema de Seguimiento y Evaluación de la Protección Social. Bogotá.

Monestier, M. (1999). Los niños esclavos. El infierno diario de trescientos millones de niños. Madrid: Alianza.

Organización Internacional del Trabajo (OIT). (1973). La edad mínima de admisión al empleo. Convenio $n^{\circ} 138$ de la Conferencia: 58.

Organización Internacional del Trabajo (OIT). (2002). Trabajo digno: una meta común de la juventud y los sindicatos.

Organización Internacional del Trabajo (OIT). (2006). Promoviendo el trabajo decente en las Américas: agenda hemisférica 2006-2015.

Organización Internacional del Trabajo (OIT). (2006). La fin du travail des enfants: un objectif à notre portée. Genève.

Organización Internacional del Trabajo (OIT). (2007). Diálogo social: todos juntos por el trabajo decente. Ginebra. 
Organización Internacional del Trabajo (OIT). (2008). Ratification et promotion des conventions fondamentales de l'OIT. Ginebra.

Organización Internacional del Trabajo (OIT). (2015) Informe mundial de 2015 sobre el trabajo infantil. Allanar el camino hacia el trabajo decente para los jóvenes. Oficina Internacional del Trabajo. Ginebra.

Organización Internacional del Trabajo (OIT) - Cinterfor. (2002). Trabajo decente y formación para jóvenes. Montevideo.

Pedraza, A.; Ribero, R. (2006). El trabajo infantil y juvenil en Colombia y algunas de sus consecuencias claves. Revista Latinoamericana de Ciencias Sociales, Niñez y Juventud, 4(1). Recuperado de: https://dialnet.unirioja.es/descarga/ articulo/4657561.pdf

Salazar, M. C. (1999). Las políticas públicas. Bogotá: Pontificia Universidad Javeriana.

Salazar, M. C. (1999). Trabajo infantil y educación en América Latina: algunos avances y efectos de las políticas educativas en la década de 1990. En: E. García y M. C. Salazar (comp.) Nuevas perspectivas para erradicar el trabajo infantil en América Latina. Bogotá: Tercer Mundo Editores.

Salazar, M.C. (2006). Los esclavos invisibles: autoritarismo, explotación y derechos de los niños en América Latina. Tunja: Universidad Pedagógica y Tecnológica de Colombia.

Tomaševski, K. (2001). Human rights obligations: making education available, accessible, acceptable and adaptable. Gotemburgo: Swedish International Development Cooperation Agency.

Umaña, E. (2002). El niño. Menores de edad. Bogotá: Unibiblos.

Unicef. (1995). Derecho a tener Derecho. Infancia, derecho y políticas sociales en América Latina y el Caribe. Tomo IV.

Zacharie, A.; Seron, A. (2008). Mondialiser le travail décent. Bruxelles: CNCD-Luc Pire. 